\title{
Clinical Comparison of 5mm Mini-incision Laparoendoscopic Single-Site Hysterectomy and Traditional Laparoendoscopic Single-Site Hysterectomy
}

\author{
Ying Cao, Yilin Sun, Jiming Chen", Zhiyong Dong, Yafeng Zheng, Yunfen Jiang, Ruxia Shi \\ Department of Obstetrics and Gynecology, the Affiliated Changzhou No. 2 People's Hospital of Nanjing Medical University, Changzhou, \\ China
}

\author{
Email address: \\ cjming@126.com (Jiming Chen) \\ ${ }^{*}$ Corresponding author
}

\section{To cite this article:}

Ying Cao, Yilin Sun, Jiming Chen, Zhiyong Dong, Yafeng Zheng, Yunfen Jiang, Ruxia Shi. Clinical Comparison of 5mm Mini-incision Laparoendoscopic Single-Site Hysterectomy and Traditional Laparoendoscopic Single-Site Hysterectomy. Journal of Gynecology and Obstetrics. Vol. 9, No. 1, 2021, pp. 21-25. doi: 10.11648/j.jgo.20210901.15

Received: February 9, 2021; Accepted: February 19, 2021; Published: February 27, 2021

\begin{abstract}
Objective: To investigate the feasibility of $5 \mathrm{~mm}$ mini-incision Laparoendoscopic Single-Site hysterectomy and Laparoendoscopic Single-Site hysterectomy in terms of safety, postoperative pain and cosmetic effect. Methods: The medical records of 19 patients who underwent hysterectomy with $5 \mathrm{~mm}$ mini-incision Laparoendoscopic Single-Site Surgery (LESS) or traditional Laparoendoscopic Single-Site Sugery in the Affiliated Changzhou No. 2 People's Hospital of Nanjing Medical University from December 2017 to June 2020 were retrospectively analyzed, including 6 cases in the group of $5 \mathrm{~mm}$ mini-incision Laparoendoscopic Single-Site hysterectomy through umbilical ordinary incision $(5 \mathrm{~mm})$ and 13 cases in the group of traditional Laparoendoscopic Single-Site hysterectomy through umbilical ordinary incision $(1.5 \mathrm{~cm}-3 \mathrm{~cm})$. Collect and compare the intraoperative blood loss, the operative time, the postoperative hemoglobin descender, the postoperative hospital stays, the probability of postoperative wound infection, the total cost, Visual analogue scale (VAS) of 12 hours after surgery, VAS of 24 hours after surgery and postoperative Body Image Questionnaire (BIQ, including Body Image scale, BIS and Cosmetic Score, CS) in the two groups. Results: There was no intraoperative complication happened, and there was no significant difference between the two groups in the forms of the intraoperative blood loss, operative time, postoperative hospital stays, postoperative hemoglobin descender, the probability of postoperative wound infection, the total cost, VAS of 24 hours after surgery and CS $(\mathrm{P}>0.05)$. But the VAS of 12 hours after surgery of the $5 \mathrm{~mm}$ mini-incision Laparoendoscopic Single-Site hysterectomy was lower than traditional Laparoendoscopic Single-Site hysterectomy (1.17 \pm 0.14 vs. $2.33 \pm 0.19)$, the BIS of the former was higher than the later $(19.83 \pm 0.15 \mathrm{vs} 19.16 \pm 0.28)$, and importantly the difference was statistically significant $(\mathrm{P}<$ 0.05). Conclusion: Laparoendoscopic Single-Site through $5 \mathrm{~mm}$ mini-incision is safe and feasible. Compared with traditional Laparoendoscopic Single-Site Hysterectomy through ordinary incision, it can not only significantly reduce postoperative pain, but also bring more satisfactory postoperative cosmetic effect.
\end{abstract}

Keywords: $5 \mathrm{~mm}$ Mini-incision, Laparoendoscopic Single-Site Surgery, Hysterectomy

\section{Introduction}

With the progress of science and technology and its application in medicine, laparoscopy has been popularized in gynecological surgery. In recent years, Laparoendoscopic Single-Site Surgery (LESS) has been rapidly developed, which further amplify the advantages of laparoscopy, such as small trauma, quick recovery and light postoperative pain. The feasibility of LESS have been confirmed by a large number of studies, Robotic LESS and vaginal natural orifice transluminal endoscopic surgery (vNOTES) [1-5]. However, there are also some scholars who believe that LESS has no obvious advantages over traditional porous laparoscopy [6, 7]. In addition, with the improvement of living standards, the majority of women have higher and higher requirements for scarless treatment. Dunker et al even designed Body image 
questionnaire (BIQ) Score, including Body Image Scale (BIS) and Cosmetic Score (CS), to compare the Cosmetic effects of minimally invasive surgery and laparotomy for Crohn's disease [8], which also puts forward higher requirements for the cosmetic effect of traditional LESS. Experts of LESS domestic and overseas are also continuously reducing surgical scar and improving the cosmetic effect as much as possible. In 2020, Marchand G et al. reported a case of laparoendoscopic single-site hysterectomy with an incision length of $11 \mathrm{~mm}$, which is the smallest reported laparoendoscopic single-site hysterectomy with a good surgical effect [9]. The department of gynecology in our hospital has performed laparoendoscopic single-site hysterectomy through a $5 \mathrm{~mm}$ mini-incision for some patients with suitable conditions since April 2018, and reported the laparoendoscopic single-site hysterectomy through a $5 \mathrm{~mm}$ mini--incision for the first time in 2019 .

\section{Method}

\subsection{General Information}

\subsubsection{Basic Information}

A total of 6 patients who received laparoendoscopic single-site hysterectomy through a $5 \mathrm{~mm}$ mini-cut in Department of Obstetrics and Gynecology, the Affiliated Changzhou No. 2 People's Hospital of Nanjing Medical University from December 2017 to June 2020 were selected as the observation group, and 13 patients who received laparoendoscopic single-site hysterectomy through ordinary incision $(1.5 \mathrm{~cm}-3 \mathrm{~cm})$ during the same period were selected as the control group. Patients of the observation group was 40-52 years old, and the median age was 51 years old. Among them, 2 patients suffered from grade III cervical intraepithelial neoplasia (CINIII), 1 patient suffered from grade I cervical intraepithelial neoplasia (CINI), 2 patients suffered from stage IA1 cervical squamous cell carcinoma, and 1 patient suffered from endometrial atypical hyperplasia. The age of the control group range from 38 to 76 years old (median age was 49 years old), among them, 5 patients suffered from atypical endometrial hyperplasia, 1 patient suffered from grade III cervical high intraepithelial lesion, 1 patient suffered from endometrial carcinoma, and 6 patients suffered from myoma of uterus or adenomyosis. All patients had no fertility requirements.

\subsubsection{Inclusion Criteria}

Patients with uniform body shape, and BMI $<30 \mathrm{~kg} / \mathrm{m}^{2}$; patients with stable vital signs and normal cardiopulmonary function, without contraindications for laparoscopic and hysteroscopic surgery; patients whose uterus was not bigger than 10 weeks of gestation.

\subsubsection{Exclusion Criteria}

Patients without normal spine and pelvis, who were unable to adopt bladder lithotripsy position; obesity patients with abdominal wall hypertrophy and puncture difficulty; patients with severe cardiopulmonary dysfunction or having taken long-term anticoagulant therapy; patients with endometriosis and other serious pelvic adhesions; patients with a history of umbilical hernia; patients whose uterus was larger than 10 weeks of gestation; Other obvious contraindications for laparoscopic surgery were excluded.

\subsection{Surgical Methods}

laparoendoscopic single-site hysterectomy through the ordinary incision in the center of the navel take about $1.5-3 \mathrm{~cm}$ long longitudinal incision, cut the skin, subcutaneous tissue, peritoneal, place the retractor disposable through the incision and support it, disposable gloves wrist set on the incision retractor and fixed seal, cut a small mouth on each finger-cot, put trocars through the finger-cots (middle finger placed 10 $\mathrm{mm}$ trocar, next to two fingers with two $5 \mathrm{~mm}$ trocar respectively), filling the $\mathrm{CO} 2$ pneumoperitoneum, keep intra-abdominal pressure between $10 \sim 12 \mathrm{mmHg}$. The middle $10 \mathrm{~mm}$ Trocar is used to place the laparoscope and connect the pneumoperitoneum machine. Two $5 \mathrm{~mm}$ Trocars are used to place the operating forceps for surgical operations, and an uterine lift was placed inside the uterine cavity to facilitate manipulation of the uterus. For $5 \mathrm{~mm}$ mini-incision laparoendoscopic single-site hysterectomy, a longitudinal incision of $5 \mathrm{~mm}$ in length was taken from the middle of the umbilical cord, which was entered into the abdomen in the same way to form pneumoperitoneum. The $5 \mathrm{~mm}$ Trocar in the middle was used to place the pediatric laparoscope, and the other two $5 \mathrm{~mm}$ Trocars next to it were used to place the operating forceps for surgical operation.

\subsection{Observation Indexes and Collection Methods}

Observation indexes including pelvic adhesion, intraoperative complications, intraoperative blood loss, operative time, postoperative hemoglobin descender, postoperative hospital stays, the rate of postoperative wound infection, the total cost, VAS of 12 hours after operation, VAS of 24 hours after operation, postoperative Body Image Questionnaire, BIQ (including Body Image scale, BIS and Cosmetic Score, CS) etc.

The perioperative information was collected by the medical record writing system, and the VAS, BIS and CS were collected during hospitalization or followed up by telephone.

\subsection{Statistical Methods}

SPSS 18.0 software was used for data processing. Measurement data was expressed as mean \pm standard deviation, analyzed by t-test. And counting data was expressed as percentage, analyzed by Fisher's exact test. P value $<0.05$ was considered statistically significant.

\section{Result}

\subsection{General Clinical Data}

Two groups of a total of 19 patients were included in the study. There was no significant difference of the observation group $(5 \mathrm{~mm}$ mini--incision laparoendoscopic single-site 
hysterectomy) and control group (traditional laparoendoscopic single-site hysterectomy) in terms of BMI, age, pregnant times, delivery times, hypertension and diabetes

mellitus. The production times and history of abdominal surgery of the observation group was less than the observation group, meaningful differences (table 1).

Table 1. Comparison of general clinical data between the two groups.

\begin{tabular}{|c|c|c|c|}
\hline Project & The observation group & The control group & Pvalue $^{*}$ \\
\hline BMI $\left(\mathrm{kg} / \mathrm{m}^{2}\right)$ & $26.27 \pm 0.935$ & $22.45 \pm 1.17$ & 0.099 \\
\hline age & $47.60 \pm 1.90$ & $50.5 \pm 2.70$ & 0.230 \\
\hline Pregnant times & $3.00 \pm 0.23$ & $2.78 \pm 0.44$ & 0.638 \\
\hline Ddelivery times & $1.00 \pm 0.00$ & $1.67 \pm 0.19$ & 0.025 \\
\hline Underwent surgery or not & & & 0.034 \\
\hline No & $6(100.0 \%)$ & $6(46.2 \%)$ & \\
\hline With hypertension & & & 0.348 \\
\hline No & $5(83.3 \%)$ & $8(61.5 \%)$ & \\
\hline Yes & $1(16.7 \%)$ & $5(35.5 \%)$ & \\
\hline With diabetes mellitus & & & 0.111 \\
\hline No & $6(100 \%)$ & $8(61.5 \%)$ & \\
\hline Yes & $0(0.0 \%)$ & $5(35.5 \%)$ & \\
\hline
\end{tabular}

(*: T-test was used for measurement data, Fisher exact test was used for counting data, and $\mathrm{P}$ value $<0.05$ was considered significant difference)

\subsection{Perioperative Results}

There were no intraoperative complications in all patients, and there were no obvious difference in pelvic adhesion degree, operative time, intraoperative blood loss, postoperative hemoglobin decline, postoperative hospital stays, postoperative infection rate, total cost and so on between the two groups (Table 2).

Table 2. Comparison of observation and treatment between the two groups.

\begin{tabular}{lll}
\hline project & The observation group & The control group \\
\hline operative time (min) & $161.67 \pm 6.44$ & $135.00 \pm 14.53$ \\
intraoperative blood loss (ml) & $45.83 \pm 8.15$ & $40.00 \pm 6.07$ \\
postoperative hemoglobin decline $(\mathrm{g} / \mathrm{L})$ & $15.33 \pm 1.55$ & $10.50 \pm 3.47$ \\
postoperative hospital stays $(\mathrm{d})$ & $13.00 \pm 2.43$ & $13.00 \pm 0.86$ \\
VAS of 12 hours after surgery & $1.17 \pm 0.14$ & $2.33 \pm 0.19$ \\
VAS of 24 hours after surgery & $0.67 \pm 0.19$ & $0.83 \pm 0.15$ \\
BIS & $19.83 \pm 0.15$ & $19.16 \pm 0.28$ \\
CS & $23.00 \pm 0.58$ & $21.83 \pm 0.75$ \\
Total cost (Yuan) & $29813.60 \pm 3071.54$ & $27316.33 \pm 1642.55$ \\
Pelvic adhesion & & 0.328 \\
without & $0(0.0 \%)$ & 0.001 \\
mild & $6(100.0 \%)$ & 0.011 \\
moderate & $0(0.0 \%)$ & 0.384 \\
Postoperative infection & & 0.566 \\
No & $6(100 \%)$ & 0.132 \\
Yes & $0(0.0 \%)$ & $4(53.8 \%)$ \\
\hline
\end{tabular}

(*: T-test was used for measurement data, Fisher exact test was used for counting data, and $\mathrm{P}$ value $<0.05$ was considered significant difference)

\subsection{Visual Analogue Scale}

VAS of 12 hours after surgery of the observation group was lower than that of the control group $(1.17 \pm 0.14$ points vs $2.33 \pm 0.19$ points), and the difference was statistically significant $(\mathrm{P}<0.05)$. VAS of 24 hours after surgery of the observation group was also lower than that of the control group $(0.67 \pm 0.19$ vs $0.83 \pm 0.15)$, but the difference make no sense $(\mathrm{P}>0.05)$. (Table 2$)$.

\subsection{Beauty Satisfaction}

Postoperative BIS score in the observation group was higher than that in the control group $(19.83 \pm 0.15$ vs $19.16 \pm 0.28)$, the difference was meaningful $(\mathrm{P}<0.05)$, suggesting that the body satisfaction and confidence of the observation group was significantly higher than that in the control group. Postoperative CS showed no significant difference between the two groups $(23.00 \pm 0.58$ vs $21.83 \pm 0.75$, $\mathrm{P}>0.05$ ). (Table 2).

\section{Discussion}

At present, most domestic and foreign studies on laparoendoscopic single-site surgery are based on the comparison between traditional laparoscopy surgery and 
laparoendoscopic single-site surgery. LESS is widely used in various surgical disciplines [10-12], and some studies have shown that LESS has the advantages of alleviating postoperative pain, shortening average hospital stays and better cosmetic effect compared with traditional laparoscopy $[13,14]$. However, some studies suggested that traditional LESS has no obvious advantages compared with traditional laparoscopy [6, 7]. The study of Sangnier et al believed that there was no significant difference between LESS and traditional laparoscopy in terms of postoperative pain, but experienced operators can shorten the operation time by LESS [6]. Reviewing the development of laparoscopic surgery, it can be seen that the direction and goal of the continuous development of LESS is to ensure or even make the minimally invasive advantages of laparoscopic surgery more obvious while enlarging surgical indications and completing more difficult and complex operations. For LESS, fewer incision and smaller incision diameter can reflect the advantages of beauty and minimally invasive. The $5 \mathrm{~mm}$ mini-incision Laparoendoscopic Single-Site Surgery is not only a further exploration on the basis of traditional LESS, but also a new technology with higher pursuit of minimally invasive and "scar invisible" effect. In this study, The $5 \mathrm{~mm}$ mini-incision Laparoendoscopic Single-Site Surgery was compared with traditional LESS in terms of safety, postoperative pain and cosmetic satisfaction, etc. On the basis of LESS, the incision size was further reduced to bring better cosmetic effect and increased the difficulty of surgery at the same time.

In this study, there was no significant difference in general clinical characteristics between the two groups except for the birth times and the history of transabdominal surgery, and the observational indicators were comparable to a certain extent. There was no complication in all operations, and the VAS of 12 hours after surgery in the observation group was lower than that in the control group ( $1.17 \pm 0.14$ vs $2.33 \pm 0.19)$, with statistical significance $(\mathrm{P}<0.05)$. The VAS of 24 hours after surgery of observation group was also lower than that of the control group $(0.67 \pm 0.19$ vs $0.83 \pm 0.15)$, but the difference was pointless $(\mathrm{P}>0.05)$. The postoperative BIS of the observation group was higher than that of the control group (19.83 \pm 0.15 vs $19.16 \pm 0.28)$, and the difference was pointful $(\mathrm{P}<0.05)$, indicating that the body satisfaction and confidence of the observation group were significantly higher than that of the control group. There was no significant difference in postoperative CS between the two groups $(23.00 \pm 0.58$ vs $21.83 \pm 0.75, \mathrm{P}>0.05)$. There were no significant difference between the two groups in operative time, intraoperative blood loss, post operative hemoglobin decline, postoperative hospital stays, postoperative infection rate and total cost. All operations were performed by the same surgeon, so the bias caused by the difference in surgical proficiency was small. This indicates that the $5 \mathrm{~mm}$ mini-incision Laparoendoscopic Single-Site Surgery is safe and feasible, as long as strict screening and the surgeons are skilled enough in LESS, the $5 \mathrm{~mm}$ mini-incision Laparoendoscopic Single-Site Surgery will not increase the risk of surgery such as bleeding, peripheral organ injury and so on.
The Body Image Questionnaire (BIQ) was designed by Dunker et al. to compare the cosmetic effects of laparoscopic and open surgery for Crohn's disease, including two parts of Body Image Scale (BIS) and Cosmetic Score (CS) [8]. This questionnaire was based on patients and compared with Manchester or Vancouver scar scores without medical workers personally inspecting scars, facilitating postoperative follow-up. It has been used in many foreign studies to evaluate the cosmetic effects of LESS and traditional laparoscopic cholecystectomy, adrenal resection, appendectomy, rectal resection, hysterectomy, etc. [15]. In this study, BIQ was also used to objectively evaluate the difference in postoperative cosmetic satisfaction between the two groups. BIS in the $5 \mathrm{~mm}$ mini-incision Laparoendoscopic Single-Site hysterectomy group was significantly higher than the other one, while postoperative CS showed no significant difference between the two groups. But BIS and CS are not uniform, which may be related to the patient's recall bias.

The disadvantages of this study are as follows: Firstly, this study is a single-center study with a small sample size; Secondly, it is a retrospective study with recall bias. Moreover, since the time of postoperative follow-up was not unified in this study, there may be bias in the postoperative time of different patients receiving follow-up. Thirdly, the amount of intraoperative blood loss in this study was estimated by the surgeon, which may be discrepant with the actual amount of blood loss. Nevertheless, this study also confirmed the feasibility and safety of the $5 \mathrm{~mm}$ mini-incision Laparoendoscopic Single-Site hysterectomy, especially for the physicians who have rich experience in common incision single-port laparoscopic surgery, it will not significantly prolong the operative time or add intraoperative blood loss. In addition, BIQ was adopted in this study to evaluate postoperative beauty satisfaction in a more scientific and objective way, further demonstrating that micro-incision laparoscopy brings better cosmetic effect. Whether there are other advantages or disadvantages of the $5 \mathrm{~mm}$ mini-incision Laparoendoscopic Single-Site surgery needs to be confirmed in a larger sample, multi-center prospective study.

\section{Conclusion}

Laparoendoscopic Single-Site through $5 \mathrm{~mm}$ mini-incision is safe and feasible. Compared with traditional Laparoendoscopic Single-Site Hysterectomy through ordinary incision, it can not only significantly reduce postoperative pain, but also bring more satisfactory postoperative cosmetic effect.

\section{Funding}

This work was supported by grants from the Scientific Research Support Program for Postdoctoral of Jiangsu Province (2019K064), the Major Science and Technology Program of Changzhou Health and Family Planning Commission (ZD201812), the Scientific Research Support 
Program for "333 Project" of Jiangsu Province (BRA2019161).

\section{References}

[1] Huang, L., et al., Comparative analysis of vaginal natural orifice transluminal endoscopic surgery versus transumbilical laparoendoscopic single-site surgery in ovarian cystectomy. J Obstet Gynaecol Res, 2021. 47 (2): p. 757-764.

[2] Ma, J., et al., The Learning Curve of Laparoendoscopic Single-Site Surgery in Benign Gynecological Diseases. J Invest Surg, 2021: p. 1-8.

[3] Misal, M., P. M. Magtibay and J. Yi, Robotic LESS and Reduced-Port Hysterectomy Using the da Vinci SP Surgical System: A Single-Institution Case Series. J Minim Invasive Gynecol, 2020.

[4] Wang, Q., et al., Stepwise Laparoendoscopic Single-site Pectopexy for Pelvic Organ Prolapse. J Minim Invasive Gynecol, 2020.

[5] Zhu, C., et al., Comparison of Laparoendoscopic Single-Site and Laparoscopic Radical Cystectomy for Male Patients with Bladder Cancer: Perioperative and Oncologic Outcomes. J Laparoendosc Adv Surg Tech A, 2021.31 (1): p. 90-94.

[6] Sangnier, E., et al., Single port laparoscopy (SPL): Retrospective study evaluating postoperative pain in comparison with conventional laparoscopy (CL). J Gynecol Obstet Hum Reprod, 2018. 47 (8): p. 365-369.

[7] Michener, C. M., et al., Meta-analysis of Laparoendoscopic Single-site and Vaginal Natural Orifice Transluminal Endoscopic Hysterectomy Compared with Multiport Hysterectomy: Real Benefits or Diminishing Returns? J Minim Invasive Gynecol, 2020.
[8] Dunker, M. S., et al., Cosmesis and body image after laparoscopic-assisted and open ileocolic resection for Crohn's disease. Surg Endosc, 1998. 12 (11): p. 1334-40.

[9] Marchand, G. J., et al., Single Port $11 \mathrm{~mm}$ Laparoscopic Hysterectomy Performed on a 32-Year-Old Female with Adhesive Disease. Case Rep Obstet Gynecol, 2020. 2020 (1-4): p. 3757391 .

[10] Wei, Y., et al., Laparoendoscopic radical prostatectomy (LRP): stepwise transition from multi-site to single-site with the aid of the transurethral port. Int Urol Nephrol, 2021. 53 (2): p. 249-255.

[11] Tsai, K. Y., et al., Long-term and short-term surgical outcomes of single-incision laparoscopic hepatectomy on anterolateral liver segments. Surg Endosc, 2020. 34 (7): p. 2969-2979.

[12] Zaccaria, L., et al., Long-Term Follow-Up of Laparoendoscopic Single-Site Partial Nephrectomy for Nonfunctioning Moieties of Renal Duplication and Fusion Anomalies in Infants and Children. J Endourol, 2020. 34 (2): p. 134-138.

[13] Demirayak, G., et al., Comparison of laparoendoscopic single-site (LESS) surgery and conventional multiport laparoscopic (CMPL) surgery for hysterectomy: long-term outcomes of abdominal incisional scar. J Obstet Gynaecol, 2020. 40 (2): p. 217-221.

[14] Chen, W., et al., Comparison of Cosmetic Effect and Pain Reduction Outcomes of Modified Mini-Laparoscopy Versus Laparoendoscopic Single-Site Surgery for Adrenalectomy. J Laparoendosc Adv Surg Tech A, 2019. 29 (12): p. 1544-1548.

[15] Song, T., et al., Cosmetic outcomes of laparoendoscopic single-site hysterectomy compared with multi-port surgery: randomized controlled trial. J Minim Invasive Gynecol, 2013. 20 (4): p. 460-7. 